\title{
Radiative corrections to the Casimir effect for the massive scalar field
}

\author{
F. A. Barone ${ }^{1}$ R. M. Cavalcanti, ${ }^{2}$ and C. Farina ${ }^{3}$ \\ Instituto de Física, Universidade Federal do Rio de Janeiro \\ Caixa Postal 68528, 21941-972 Rio de Janeiro, RJ
}

\begin{abstract}
We compute the $O(\lambda)$ correction to the Casimir energy for the massive $\lambda \phi^{4}$ model confined between a pair of parallel plates. The calculations are made with Dirichlet and Neumann boundary conditions. The correction is shown to be sensitive to the boundary conditions, except in the zero mass limit, in which case our results agree with those found in the literature.
\end{abstract}

Since the publication of Casimir's paper in 1948 [1] on the attraction of two neutral, parallel and perfectly conducting plates (an effect that bears his name since then), a lot of work on this subject has been done [2]. However, the great majority of papers concerning the Casimir effect found in literature deals with non-interacting fields. Quite a few papers are devoted to radiative corrections to the Casimir effect (in the context of QED, see 2, 3]; in the context of the $\lambda \phi^{4}$ theory, see [4, 5]). For free fields, the Casimir energy is given only by the zero-point energy of the field, properly regularized and renormalized. Hence, different boundary conditions leading to the same eigenfrequencies yield the same Casimir energy. In other words, at one-loop level the Casimir effect is sensitive to the eigenfrequencies, but blind to the eigenmodes. An interesting question is whether this peculiarity is maintained if radiative corrections are taken into account. In the case of a massless scalar field with a quartic self-interaction confined between a pair of parallel plates, it has been shown that the first order radiative correction to the Casimir energy is the same for Dirichlet and Neumann boundary conditions [4]. However, it is not clear whether this is also true in the case of a massive scalar field or is a peculiarity of the massless field. Even for the massless scalar field, it is not obvious whether this property will persist at higher orders in the coupling constant. The purpose of this work is to investigate the former question, namely, what is the effect of the mass on the first order radiative correction to the Casimir energy of a self-interacting scalar field confined between a pair of parallel plates.

\footnotetext{
${ }^{1}$ Email: fabricio@if.ufrj.br

${ }^{2}$ Email: rmoritz@if.ufrj.br

${ }^{3}$ Email: farina@if.ufrj.br
} 
We shall consider in this paper the massive $\lambda \phi^{4}$ model in four-dimensional space-time. It is defined by the Euclidean Lagrangian density ${ }^{4}$

$$
\mathcal{L}_{\mathrm{E}}=\frac{1}{2}\left(\partial_{\mu} \phi\right)^{2}+\frac{1}{2} m^{2} \phi^{2}+\frac{\lambda}{4 !} \phi^{4}+\mathcal{L}_{\mathrm{CT}},
$$

where $\mathcal{L}_{\mathrm{CT}}$ contains the usual renormalization counterterms. The system is confined between two parallel plates, located at the planes $z=0$ and $z=a$, where the field is submitted to one of the following boundary conditions:

- Dirichlet boundary conditions (DD):

$$
\phi(z=0)=\phi(z=a)=0
$$

- Neumann boundary conditions (NN):

$$
\left.\frac{\partial \phi}{\partial z}\right|_{z=0}=\left.\frac{\partial \phi}{\partial z}\right|_{z=a}=0
$$

In the noninteracting case (i.e., $\lambda=0$ ), the Casimir energy per unit of area is the same for both kinds of boundary conditions [6]:

$$
E_{\mathrm{DD}}^{(0)}=E_{\mathrm{NN}}^{(0)}=-\frac{m^{2}}{16 \pi^{2} a} \sum_{n=1}^{\infty} \frac{K_{2}(2 a m n)}{n^{2}} .
$$

Using perturbation theory, one obtains for the $O(\lambda)$ correction to the above result the following expression:

$$
E^{(1)}=\int_{0}^{a} d z\left[\frac{\lambda}{8} G^{2}(x, x)+\frac{\delta m^{2}}{2} G(x, x)+\delta \Lambda\right],
$$

where $G\left(x, x^{\prime}\right)$ is the Green's function of the free theory (i.e., with $\lambda=0$, but obeying the boundary conditions), $\delta m^{2}$ is the radiatively induced shift in the mass parameter, and $\delta \Lambda$ is the shift in the cosmological constant (i.e., the change in the vacuum energy which is due solely to the interaction, and not to the confinement).

In the spectral representation, the Green's function in $(d+1)$-dimensional space-time is given by

$$
G\left(x, x^{\prime}\right)=\int \frac{d \omega}{2 \pi} \int \frac{d^{d-1} k}{(2 \pi)^{d-1}} e^{-i \omega\left(\tau-\tau^{\prime}\right)+i \mathbf{k} \cdot\left(\mathbf{r}-\mathbf{r}^{\prime}\right)} \sum_{n} \frac{\varphi_{n}(z) \varphi_{n}^{*}\left(z^{\prime}\right)}{\omega^{2}+k^{2}+m^{2}+(n \pi / a)^{2}},
$$

\footnotetext{
${ }^{4}$ Conventions: $\hbar=c=1, x=(\tau, \mathbf{r}, z)$.
} 
with

$$
\begin{aligned}
\varphi_{n}^{\mathrm{DD}}(z) & =\sqrt{\frac{2}{a}} \sin \left(\frac{n \pi z}{a}\right), \quad n=1,2,3, \ldots \\
\varphi_{n}^{\mathrm{NN}}(z) & =\sqrt{\frac{2-\delta_{n, 0}}{a}} \cos \left(\frac{n \pi z}{a}\right), \quad n=0,1,2,3, \ldots
\end{aligned}
$$

$G\left(x, x^{\prime}\right)$ diverges when $x^{\prime} \rightarrow x$ for $d \geq 1$, therefore a regularization prescription is needed in order to make sense of $G(x, x)$. We shall compute it using dimensional regularization; the result is

$$
G(x, x)=\frac{\Gamma(1-d / 2)}{(4 \pi)^{d / 2}} \sum_{n} \omega_{n}^{d-2} \varphi_{n}(z) \varphi_{n}^{*}(z) \quad(d<1)
$$

where

$$
\omega_{n}=\sqrt{m^{2}+\left(\frac{n \pi}{a}\right)^{2}}
$$

Now we can compute the terms appearing in Eq. (15):

$$
\begin{gathered}
\int_{0}^{a} d z G(x, x)=\frac{\Gamma(1-d / 2)}{(4 \pi)^{d / 2}} \sum_{n=n_{0}}^{\infty} \omega_{n}^{d-2}, \\
\int_{0}^{a} d z G^{2}(x, x)=\frac{\Gamma^{2}(1-d / 2)}{4 \pi a}\left[\left(\sum_{n=n_{0}}^{\infty} \omega_{n}^{d-2}\right)^{2}+\frac{1}{2} \sum_{n=1}^{\infty} \omega_{n}^{2 d-4}\right],
\end{gathered}
$$

where $n_{0}=1$ in the DD case, and $n_{0}=0$ in the NN case.

Collecting terms, we obtain

$$
\begin{aligned}
E_{\mathrm{DD}}^{(1)}= & \frac{\lambda}{8 a}\left[\frac{\Gamma(1-d / 2)}{(4 \pi)^{d / 2}} F(2-d)+\frac{2 a \delta m^{2}}{\lambda}\right]^{2} \\
& +\frac{\lambda}{16 a} \frac{\Gamma^{2}(1-d / 2)}{(4 \pi)^{d}} F(4-2 d)+\left[\delta \Lambda-\frac{\left(\delta m^{2}\right)^{2}}{2 \lambda}\right] a, \\
E_{\mathrm{NN}}^{(1)}= & \frac{\lambda}{8 a}\left[\frac{\Gamma(1-d / 2)}{(4 \pi)^{d / 2}}\left[F(2-d)+m^{d-2}\right]+\frac{2 a \delta m^{2}}{\lambda}\right]^{2} \\
& +\frac{\lambda}{16 a} \frac{\Gamma^{2}(1-d / 2)}{(4 \pi)^{d}} F(4-2 d)+\left[\delta \Lambda-\frac{\left(\delta m^{2}\right)^{2}}{2 \lambda}\right] a,
\end{aligned}
$$

where $F(s)$ is defined as

$$
F(s) \equiv \sum_{n=1}^{\infty} \omega_{n}^{-s}=\sum_{n=1}^{\infty}\left[m^{2}+\left(\frac{n \pi}{a}\right)^{2}\right]^{-s / 2}, \quad \Re(s)>1 .
$$

The identity [6]

$$
F(s)=-\frac{1}{2} m^{-s}+\frac{a m^{1-s}}{2 \pi^{1 / 2} \Gamma(s / 2)}\left[\Gamma\left(\frac{s-1}{2}\right)+4 \sum_{n=1}^{\infty} \frac{K_{(1-s) / 2}(2 a m n)}{(a m n)^{(1-s) / 2}}\right],
$$


where $K_{\nu}$ denotes the modified Bessel function, provides the analytic extension of $F(s)$ to $\Re(s) \leq 1$, with simple poles at $s=1,-1,-3,-5, \ldots$

Let us now fix the renormalization conditions for $\delta m^{2}$ and $\delta \Lambda$. To do so, let us consider the self-energy $\Sigma$. In first order in perturbation theory it is given by

$$
\Sigma(x)=\frac{\lambda}{2} G(x, x)+\delta m^{2} .
$$

$\delta m^{2}$ will be fixed by imposing the following conditions on $\Sigma(x)$ : (i) that $\Sigma(x)<\infty$ (except possibly at some special points), and (ii) that $\Sigma(x)$ vanishes away from the plates when $a \rightarrow \infty$, i.e.,

$$
\lim _{a \rightarrow \infty} \Sigma(z=\gamma a)=0, \quad 0<\gamma<1
$$

besides, we shall require that $\delta m^{2}$ be independent of $a$. These conditions are fulfilled by taking $\delta m^{2}=-(\lambda / 2) G_{0}(0)$, where $G_{0}(z)$ denotes the free Green's function without boundary conditions evaluated at the point $x=(0, \mathbf{0}, z)$. (This is most easily seen in the multiple reflection representation of the Green's function $G$, in which Eq. (17) becomes

$$
\Sigma(x)=\frac{\lambda}{2} \sum_{n=-\infty}^{\infty}\left[G_{0}(2 n a) \pm G_{0}(2 z+2 n a)\right]+\delta m^{2},
$$

where the $+(-)$ sign corresponds to the $\mathrm{NN}(\mathrm{DD})$ case.) Computing $G_{0}(0)$ within dimensional regularization, we explicitly obtain

$$
\delta m^{2}=-\frac{\lambda}{2} \int \frac{d \omega}{2 \pi} \int \frac{d^{d} k}{(2 \pi)^{d}} \frac{1}{\omega^{2}+k^{2}+m^{2}}=-\frac{\lambda}{2} \frac{\Gamma((1-d) / 2)}{(4 \pi)^{(d+1) / 2}} m^{d-1} .
$$

For the shift in the cosmological constant we shall take

$$
\delta \Lambda=\frac{\left(\delta m^{2}\right)^{2}}{2 \lambda}
$$

With this choice one eliminates the terms proportional to $a$ in Eqs. (13) and (14), which do not contribute to the force between the plates (the linear dependence on $a$ is canceled by similar terms when one adds the energy of the regions $z<0$ and $z>a$ ).

Inserting Eqs. (16), (20), and (21) into Eqs. (13) and (14), we obtain

$$
\begin{aligned}
E_{\mathrm{DD}}^{(1)}= & \frac{\lambda}{8 a}\left[\frac{4 a m^{d-1}}{(4 \pi)^{(d+1) / 2}} \sum_{n=1}^{\infty} \frac{K_{(d-1) / 2}(2 a m n)}{(a m n)^{(d-1) / 2}}-\frac{\Gamma(1-d / 2)}{2(4 \pi)^{d / 2}} m^{d-2}\right]^{2} \\
& +\frac{\lambda}{32 a} \frac{\Gamma^{2}(1-d / 2)}{(4 \pi)^{d}}\left\{-m^{2 d-4}+\frac{a m^{2 d-3}}{\pi^{1 / 2} \Gamma(2-d)}\left[\Gamma\left(\frac{3}{2}-d\right)\right.\right. \\
& \left.\left.+4 \sum_{n=1}^{\infty} \frac{K_{(2 d-3) / 2}(2 a m n)}{(a m n)^{(2 d-3) / 2}}\right]\right\}
\end{aligned}
$$




$$
\begin{aligned}
E_{\mathrm{NN}}^{(1)}= & \frac{\lambda}{8 a}\left[\frac{4 a m^{d-1}}{(4 \pi)^{(d+1) / 2}} \sum_{n=1}^{\infty} \frac{K_{(d-1) / 2}(2 a m n)}{(a m n)^{(d-1) / 2}}+\frac{\Gamma(1-d / 2)}{2(4 \pi)^{d / 2}} m^{d-2}\right]^{2} \\
& +\frac{\lambda}{32 a} \frac{\Gamma^{2}(1-d / 2)}{(4 \pi)^{d}}\left\{-m^{2 d-4}+\frac{a m^{2 d-3}}{\pi^{1 / 2} \Gamma(2-d)}\left[\Gamma\left(\frac{3}{2}-d\right)\right.\right. \\
& \left.\left.+4 \sum_{n=1}^{\infty} \frac{K_{(2 d-3) / 2}(2 a m n)}{(a m n)^{(2 d-3) / 2}}\right]\right\} .
\end{aligned}
$$

We can now take $d=3$, obtaining

$$
\begin{aligned}
& E_{\mathrm{DD}}^{(1)}=\frac{\lambda m^{2}}{512 \pi^{2} a}\left[\left(1+\frac{2}{\pi} \sum_{n=1}^{\infty} \frac{K_{1}(2 a m n)}{n}\right)^{2}-1\right], \\
& E_{\mathrm{NN}}^{(1)}=\frac{\lambda m^{2}}{512 \pi^{2} a}\left[\left(1-\frac{2}{\pi} \sum_{n=1}^{\infty} \frac{K_{1}(2 a m n)}{n}\right)^{2}-1\right] .
\end{aligned}
$$

It follows froms Eqs. (24) and (25) that $E_{\mathrm{DD}}^{(1)} \geq E_{\mathrm{NN}}^{(1)}$ in three-dimensional space; equality is attained only in the massless case, in which case one recovers the results of Ref. [4]:5

$$
E_{\mathrm{DD}}^{(1)}=E_{\mathrm{NN}}^{(1)}=\frac{\lambda}{18432 a^{3}} \quad(d=3, m=0)
$$

It is also interesting to note that, while $E_{\mathrm{DD}}^{(1)}$ is a monotonically decreasing function of $a$, $E_{\mathrm{NN}}^{(1)}$ first decreases and then eventually increases with $a$. Indeed, Eq. (26) is also valid for fixed $m$ and $a \rightarrow 0$; on the other hand, for $a \gg m^{-1}$ Eqs. (24) and (25) yield

$$
E_{\mathrm{NN}}^{(1)} \approx-E_{\mathrm{DD}}^{(1)} \approx-\frac{\lambda m^{3 / 2}}{256 \pi^{5 / 2}} a^{-3 / 2} \exp (-2 m a)
$$

Results for other kinds of boundary conditions (DN, periodic, and anti-periodic) will be presented elsewhere [8].

\section{Acknowledgments}

This work was supported by CNPq and CAPES.

\footnotetext{
${ }^{5}$ In order to take the limit $m \rightarrow 0$ in Eqs. (24) and (25), we have used the following expansion, valid for small $z$ []:

$$
\sum_{n=1}^{\infty} \frac{K_{1}(n z)}{n}=\frac{\pi^{2}}{6 z}-\frac{\pi}{2}+O(z \ln z)
$$
}




\section{References}

[1] H. B. G. Casimir, Proc. K. Ned. Akad. Wet. 51, 793 (1948).

[2] For a recent review, see M. Bordag, U. Mohideen, and V. M. Mostepanenko, Phys. Rep. 353, 1 (2001).

[3] M. Bordag, D. Robaschik, and E. Wieczorek, Ann. Phys. (N.Y.) 165, 192 (1985); E. Wieczorek, D. Robaschik, and K. Scharnhorst, Sov. J. Nucl. Phys. 44, 665 (1986); D. Robaschik, K. Scharnhorst, and E. Wieczorek, Ann. Phys. (N.Y.) 174, 401 (1987); M. Bordag and K. Scharnhorst, Phys. Rev. Lett. 81, 3815 (1998); M. Bordag and J. Lindig, Phys. Rev. D 58, 045003 (1998); F. Ravndal and J. B. Thomassen, Phys. Rev. D 63, 113007 (2001); K. Melnikov, Phys. Rev. D 64, 045002 (2001).

[4] M. Krech and S. Dietrich, Phys. Rev. A 46, 1886 (1992).

[5] B. S. Kay, Phys. Rev. D 20, 3052 (1979); L. H. Ford, Proc. R. Soc. Lond. A 368, 305 (1979); Phys. Rev. D 22, 3003 (1980); L. H. Ford and T. Yoshimura, Phys. Lett. A 70, 89 (1979); D. J. Toms, Phys. Rev. D 21, 928 (1980); 21, 2805 (1980); Ann. Phys. (N.Y.) 129, 334 (1980); K. Symanzik, Nucl. Phys. B190, 1 (1981); L. C. de Albuquerque and R. M. Cavalcanti, Phys. Rev. D 65, 045004 (2002).

[6] J. Ambjørn and S. Wolfram, Ann. Phys. (N.Y.) 147, 1 (1983).

[7] H. W. Braden, Phys. Rev. D 25, 1028 (1982).

[8] F. A. Barone, R. M. Cavalcanti, and C. Farina, to appear. 